\title{
Sensorineural Hearing Loss and Congenital Cytomegalovirus Infection
}

\author{
Beuy Joob ${ }^{1}$ Viroj Wiwanitkit ${ }^{2}$ \\ ${ }^{1}$ Medical Center Department, Sanitation 1 Medical Academic Center, \\ Bangkok, Thailand \\ 2 Department of Tropical Medicine, Hainan Medical University, Hainan \\ Sheng, Republic of China \\ J Pediatr Genet 2018;7:45.
}

We read the article on "Sensorineural hearing loss in a patient affected by congenital cytomegalovirus infection" with a great interest. ${ }^{1}$ Fontana et al reported on "a 3-year-old female patient with symptomatic congenital cytomegalovirus (CMV) infection who also exhibited developmental delay, dysmorphic facial features, bilateral hearing loss, and cochlear incomplete partition, type 2, with 7q21.3 deletion."1 Fontana et al raised an important question, "Is it useful to identify comorbid pathologies?" In fact, there are several causes of sensorineural hearing loss. The genetic investigation might be useful for the diagnosis of possible underlying factor. However, there is no clear information on the prevalence of sensorineural hearing loss due to the genetic problem. The problem might or might not be more common than the environmental factors such as infections. ${ }^{2}$ The general screening for hearing loss might be useful in the area with high prevalence, ${ }^{3}$ but the clinical value of specific genetic test should be discussed. The clinical usefulness of the genetic investigation has to be assessed for the cost effectiveness. In our setting, in tropical
Address for correspondence Beuy Joob, PhD, Medical Center Department, Sanitation 1 Medical Academic Center, Bangkok, Thailand (e-mail: beuyjoob@hotmail.com).

country, the infection is the main cause of congenital sensorineural hearing loss, and the genetic investigation is usually not indicated.

Conflict of Interest

None.

\section{References}

1 Fontana P, Melis D, D'Amico A, et al. Sensorineural hearing loss in a patient affected by congenital cytomegalovirus infection: is it useful to identify comorbid pathologies? J Pediatr Genet 2017; 6(03):181-185

2 Korver AM, Smith RJ, Van Camp G, et al. Congenital hearing loss. Nat Rev Dis Primers 2017;3:16094 doi: 10.1038/nrdp.2016.94.

3 González-Jiménez B, Delgado-Mendoza E, Rojano-González R, et al. Neonatal Hearing Screening and Early Intervention, a screening program to evaluate all infants to identify the hearing impaired [in Spanish]. Rev Med Inst Mex Seguro Soc 2017;55(01): 40-46 received

October 31, 2017

accepted after revision

November 6, 2017

published online

December 13, 2017
Copyright @ 2018 by Georg Thieme

Verlag KG, Stuttgart · New York
DOI https://doi.org/ 10.1055/s-0037-1612599. ISSN 2146-4596. 